Research in Astronomy and Astrophysics manuscript no.

(LATEX: paper.tex; printed on July 1, 2013; 0:03)

\title{
Studies of grand minima in sunspot cycles from a flux transport solar dynamo model
}

\author{
Bidya Binay Karak and Arnab Rai Choudhuri \\ Department of Physics, Indian Institute of Science, Bangalore 560012; bidya_karak@physics.iisc.ernet.in
}

\begin{abstract}
We propose that grand minima in solar activity are caused by simultaneous fluctuations in the meridional circulation and the Babcock-Leighton mechanism for the poloidal field generation in the flux transport dynamo model. We present the following results: (a) fluctuations in the meridional circulation are more effective in producing grand minima; (b) both sudden and gradual initiations of grand minima are possible; (c) distributions of durations and waiting times between grand minima seem to be exponential; (d) the coherence time of the meridional circulation has an effect on the number and the average duration of grand minima, a coherence time of about 30 years being consistent with observational data. We also study the occurrence of grand maxima and find that the distributions of durations and waiting times between grand maxima are also exponential, like the grand minima. Finally we address the question whether the Babcock-Leighton mechanism can be operative during grand minima when there are no sunspots. We show that an $\alpha$-effect restricted to the upper portions of the convection zone can pull the dynamo out of the grand minima and can match various observational requirements if the amplitude of this $\alpha$-effect is suitably fine-tuned.
\end{abstract}

\section{INTRODUCTION}

One intriguing aspect of the solar cycle is the occurrence of grand minima when sunspots may not appear for several decades and a few cycles may go missing. Since the beginning of the telescopic observations of sunspots, one grand minimum known as the Maunder minimum occurred during 1645-1715 (Eddy 1976; Ribes \& Nesme-Ribes 1993). We have to look for indirect proxy data to infer the occurrences of grand minima at still earlier times. When the magnetic field of the Sun is weak, more cosmic rays reach the Earth's atmosphere, producing larger amounts of the cosmogenic isotopes like ${ }^{14} \mathrm{C}$ and ${ }^{10} \mathrm{Be}$. From the study of ${ }^{14} \mathrm{C}$ in old tree rings and ${ }^{10} \mathrm{Be}$ in polar ice cores, several groups have identified a number of grand minima in the past few millennia (Usoskin et al. 2007; Steinhilber et al. 2012). Particularly, Usoskin et al. (2007) have detected about 27 such events of low activity in last 11,400 years from the ${ }^{14} \mathrm{C}$ data. Even when sunspots are not seen, some of the indirect proxy data indicate the presence of continued oscillations at a subdued level during grand minima (Fligge et al. 1999). Miyahara et al. (2004) found the oscillations to have longer periods during the Maunder minimum. Miyahara et al. $(2006,2007)$ and Nagaya et al. $(2012)$ 
The aim of the present paper is to investigate whether different aspects of grand minima can be explained with a flux transport solar dynamo model. An earlier paper by Choudhuri \& Karak (2012; hereafter CK12) (also see Karak \& Choudhuri 2013) developed a theoretical model of grand minima by introducing appropriate fluctuations in our flux transport dynamo model and presented some preliminary result. This paper is a continuation of that work and addresses several aspects of the problem not discussed in CK12.

The flux transport dynamo model has emerged as the most promising theoretical model for the sunspot cycle in recent years (Wang et al. 1991; Choudhuri et al. 1995; Durney 1995; Dikpati \& Charbonneau 1999; Nandy \& Choudhuri 2002; Charbonneau 2010; Choudhuri 2011; Karak \& Petrovay 2013; Jiang et al. 2013). The primary mechanism for the poloidal field generation in this model is the Babcock-Leighton mechanism involving the decay of tilted bipolar sunspots (Babcock 1961; Leighton 1969). Since this mechanism depends on the existence of sunspots in order to be operative, this mechanism may not work during a grand minimum when there are no sunspots. We would then require some other mechanism to pull the Sun out of the grand minimum. Early models of the solar dynamo invoked the $\alpha$-effect proposed by Parker (1955) and Steenbeck et al. (1966) to generate the poloidal field. The $\alpha$-effect can twist a toroidal field to produce a poloidal field only if the toroidal field is not stronger than the equipartition field. After simulations of the buoyant rise of flux tubes suggested a much stronger toroidal field (Choudhuri \& Gilman 1987; Choudhuri 1989; D'Silva \& Choudhuri 1993; Fan et al. 1993), the flux transport dynamo models used the BabcockLeighton mechanism as the favoured mechanism rather then the $\alpha$-effect for generating the poloidal field. During a grand minimum, the toroidal field presumably becomes much weaker and probably the $\alpha$-effect can be operative to pull the Sun out of the grand minimum. Since we have very little knowledge of the nature of this $\alpha$-effect, CK12 assumed the same Babcock-Leighton mechanism to be operative all the time to simplify the theoretical calculations. One of the things we explore in this paper is the nature of the $\alpha$-effect needed to pull the dynamo out of the grand minimum. We shall see that various observational requirements put some important constraints on the nature of this $\alpha$-effect.

Let us now come to the question of what can cause irregularities in the solar cycle and the grand minima. One important question is whether the nonlinearities in the system can induce chaotic behaviour. The simplest kind of nonlinearity used extensively in the earlier dynamo models is the $\alpha$-quenching. If the magnetic field becomes stronger than usual due to some reason, this quenching makes $\alpha$ smaller and the dynamo weaker, bringing down the magnetic field. If the magnetic field becomes weaker, then the opposite happens. A nonlinearity in the form of $\alpha$-quenching makes the dynamo more stable instead of producing chaotic behaviour. A few authors have found intermittency behaviour in highly truncated dynamo models with more complicated kinds of nonlinearity which do not seem justified by solar observations (Weiss et al. 1984; Wilmot-Smith et. al. 2005).

One other source of irregularity is stochastic noise. Since the mean-field dynamo theory is obtained by averaging over turbulence, we expect turbulent fluctuations to provide a random noise. Hoyng (1988) realized this for the first time and later several authors showed that stochastic noise introduced in the mean-field dynamo equation can produce irregularities in solar cycles including grand minima-like episodes (Choudhuri 1992; Charbonneau et al. 2004; Gómez \& Mininni 2006; Brandenburg \& Spiegel 2008; Moss 
flux transport dynamo was provided by Choudhuri et al. (2007). The Babcock-Leighton mechanism for poloidal field generation depends on the tilts of bipolar sunspots. Although the average tilt at a latitude is given by Joy's law, one finds a scatter around the average (Dasi-Espuig et al. 2010), presumably due to the effect of convective turbulence on the rising flux tubes (Longcope \& Choudhuri 2002). So we believe that the Babcock-Leighton process intrinsically has a random component. Choudhuri et al. (2007) incorporated this effect allowing the poloidal field generated at the end of a cycle to differ from its average value. This approach has been followed in subsequent papers (Jiang et al. 2007; Goel \& Choudhuri 2009; Choudhuri \& Karak 2009) as well as the present paper. Choudhuri \& Karak (2009) have shown that, if the poloidal field becomes sufficiently weak at the end of a cycle due to fluctuations in the Babcock-Leighton mechanism, then that may trigger a grand minimum.

Another source of irregularities is fluctuations in meridional circulation, of which the importance has been recognized only recently (Yeates et al. 2008; Karak 2010; Karak \& Choudhuri 2011; Passos 2012). The meridional circulation plays a crucial role in the flux transport dynamo model (Choudhuri et al. 1994; Dikpati \& Charbonneau 1999; Karak 2010). Although we have neither good theoretical understanding nor long observational measurements of the meridional circulation, Karak \& Choudhuri (2011) used durations of sunspot cycles in the last 250 years to have some idea about fluctuations in meridional circulation. They concluded that the meridional circulation had large temporal variations with coherence time more than a solar cycle. There are also many other evidences for variations of the meridional circulation in past (e.g., Passos \& Lopes 2008; Passos 2012). When the meridional circulation slows down, the period of the dynamo becomes longer. This has rather different effects on dynamo models with high and low turbulent diffusivity (Yeates et al. 2008; Karak 2010; Karak \& Nandy 2012). If the turbulent diffusivity is assumed reasonably high (which is the case in our model), then the cycles become weaker because diffusivity has a longer time to act in a cycle. On the other hand, if the turbulent diffusivity is low (Dikpati \& Charbonneau 1999), then the effect of diffusivity is not so strong and the cycles become stronger with decreasing meridional circulation because the differential rotation has a longer time to act on the magnetic fields. Only a dynamo model with reasonably high turbulent diffusion (like what we use) can explain observational effects like the dipolar parity of the Sun (Chatterjee et al. 2004; Hotta \& Yokoyama 2010), the Waldmeier effect (Karak \& Choudhuri 2011), the period and the amplitude relation (Karak 2010) and the lack of significant hemispheric asymmetry (Chatterjee \& Choudhuri 2006; Goel \& Choudhuri 2009). See Section 5 of Jiang et al. (2007) and Miesch et al. (2012) (also see Muñoz-Jaramillo et al. 2013) for a discussion on this topic. In the dynamo model with high diffusivity in which a weaker meridional circulation makes cycles weaker, Karak (2010) has shown that a sufficiently weak meridional circulation can trigger a grand minimum.

Our recent paper CK12 studies the occurrence of grand minima in our theoretical dynamo model by introducing simultaneous fluctuations in the poloidal field generation and the meridional circulation. The levels of fluctuations were determined from the observational data of the last 28 cycles. With such fluctuations, the flux transport dynamo model developed in our group showed 24-30 grand minima in a typical run of 11,000 years-in close agreement with observational data.

Because of the shortness of this Letter paper CK12, a full exploration of the different aspects of the 
the model in Section 2, we present in Section 3 various aspects of the results not discussed in CK12, such as the relative importance of the two fluctuations and the dependence on parameters like the coherence time of the meridional circulation. We also present some results of grand maxima, which could not be discussed in CK12. Finally, Section 4 addresses the important question of how the dynamo comes out of the grand minimum and if we can say something about the nature of the $\alpha$-effect which may be needed for this.

\section{MODEL}

We carry out all the calculations using a flux transport dynamo model originally presented in Chatterjee et al. (2004). This model is based on the kinematic mean-field dynamo theory in which the poloidal field generation is assumed to be due to the Babcock-Leighton process. Assuming axisymmetry, the evolutions of the magnetic field components in this model are described by the following two equations:

$$
\begin{gathered}
\frac{\partial A}{\partial t}+\frac{1}{s}(\mathbf{v} \cdot \nabla)(s A)=\eta_{p}\left(\nabla^{2}-\frac{1}{s^{2}}\right) A+\alpha B \\
\frac{\partial B}{\partial t}+\frac{1}{r}\left[\frac{\partial}{\partial r}\left(r v_{r} B\right)+\frac{\partial}{\partial \theta}\left(v_{\theta} B\right)\right]=\eta_{t}\left(\nabla^{2}-\frac{1}{s^{2}}\right) B+s\left(\mathbf{B}_{p} \cdot \nabla\right) \Omega+\frac{1}{r} \frac{d \eta_{t}}{d r} \frac{\partial(r B)}{\partial r}
\end{gathered}
$$

with $s=r \sin \theta$. Here $A$ is the vector potential of the poloidal magnetic field $\left(\mathbf{B}_{\mathbf{p}}\right), B$ is the toroidal magnetic field, $\mathbf{v}=v_{r} \hat{r}+v_{\theta} \hat{\theta}$ is the meridional circulation, $\Omega$ is the internal angular velocity, $\eta_{p}, \eta_{t}$ are the turbulent diffusivities for the poloidal and toroidal components, and $\alpha$ is the source term for the poloidal field which parameterized the Babcock-Leighton mechanism. The details of all these parameters are specified in Chatterjee et al. (2004). However, Karak (2010) recently modified a few parameters slightly and in this work we are using exactly the same parameters as used in Karak (2010).

Just to remind the readers, we mention that in the expression of the meridional circulation there is a parameters $v_{0}$ which determines the strength of the meridional circulation. For normal cycle with period of 11 years we take $v_{0}=23 \mathrm{~m} \mathrm{~s}^{-1}$. However, in this work, when we introduce fluctuations in the meridional circulation, we change this $v_{0}$ to change the strength of the meridional circulation.

Let us make a comment on the absolute value of the magnetic field in our results. If the equations are completely linear in the magnetic field, then the unit of the magnetic field would be arbitrary. While (1) and (2) are linear equations, our problem becomes nonlinear when we include magnetic buoyancy following the methodology used in the earlier papers from our group (see Chatterjee et al. 2004). If the amplitude of the toroidal magnetic field $|B|$ above the bottom of the convection zone is larger than a critical value $B_{c}$, then a part of the toroidal field is made to rise to surface. This nonlinearity limits the amplitude of the magnetic field. We take $B_{c}=0.8$ which makes the maximum value of the magnetic field at the bottom of the convection zone hover around 1. Since simulations of flux tube rise based on the thin flux tube equation (Spruit 1981; Choudhuri 1990) suggest magnetic fields of the order of $10^{5} \mathrm{G}$ at the bottom of the convection zone (Choudhuri \& Gilman 1987; Choudhuri 1989; D’Silva \& Choudhuri 1993; Fan et al. 1993), it is tempting to identify the value 1 of the magnetic field in our simulations with $10^{5}$ G. However, such an identification is questionable. Apart from the fact this would give values of the polar magnetic field disagreeing with observations, we expect the magnetic field to be $10^{5} \mathrm{G}$ only inside flux tubes, whereas the dynamo equation deals with the mean magnetic field. If the filling factor of flux tubes at the bottom of the 
magnetic field computed in our model may be much less than $10^{5} \mathrm{G}$. We shall discuss these considerations further in Section 4 where we discuss whether the dynamo requires an $\alpha$-effect in addition to the BabcockLeighton mechanism to bring it out of the grand minimum.

In the next section, we present results based on exactly the same model of producing grand minima which was used in CK12. We basically discuss a few important aspects of the problem which could not be included in CK12 due to the lack of space. Then, in Section 4, we shall allow the possibility that the poloidal field generation under normal cycle conditions and during the grand minima may require different mechanisms.

\section{SIMULATIONS OF GRAND MINIMA}

Our earlier paper CK12 explained the basic assumptions of our model of grand minima and presented some illustrative results. CK12 being a letter paper, a full discussion of the results could not be presented in it. This section presents some additional results based on the grand minimum model of CK12. Since the details of the model have been given in CK12, we simply mention the salient features. The grand minima in our model were produced by fluctuations in meridional circulation and by fluctuations in the BabcockLeighton process that would make scaled polar field amplitude $\gamma$ at the end of a cycle vary from cycle to cycle. Assuming the fact that the solar cycle period is inversely related to the strength of the meridional circulation, we estimated the nature of fluctuations in the meridional circulation from the durations of the last 28 cycles. On the other hand, assuming that the solar cycle strength is directly correlated to the strength of the polar field at the end of the previous cycle, we have obtained the fluctuations in $\gamma$ from the strengths of the last 28 solar cycles. See Figure 2 of CK12 and the corresponding text for details. Assuming both the fluctuations to obey Gaussian distributions, we have constructed the distributions of these fluctuations by using the mean and the standard deviations of these data. Figure 1 shows the typical histograms of these. We then make $v_{0}$ and $\gamma$ vary randomly following these distributions. We point out that for different runs we generate different results for different realizations of the fluctuations of $v_{0}$ and $\gamma$. Another important thing to note is that we change the polar field by the factor $\gamma$ at every solar minimum whereas we change $v_{0}$ after a certain time interval called the coherence time $\tau_{M C}$. Now we shall explore the origin and different aspects of grand minima systematically.

\subsection{Contributions of meridional circulation and the poloidal field in triggering grand minima}

Using the same parameters of the basic dynamo model as used in CK12, we explore the relative importance of the contributions of the meridional circulation fluctuations and the poloidal field fluctuations in triggering grand minima. We perform two separate simulation runs by including only one kind of fluctuations in each run. First, we do a simulation by randomly varying only $v_{0}$ after intervals of 30 years (i.e., $\tau_{M C}=30$ years). The random values for $v_{0}$ used for this run have been shown in Figure1 1 a). The top panel of Figure 2 shows the results of this run. For the clarity of display we show only a small clip of 1000 years long data. Next, we present a simulation with only poloidal field fluctuations. We change the poloidal field factor $\gamma$ at every solar minimum. The histogram of $\gamma$ for this run is shown in Figure 1 b), whereas the results of this run is 

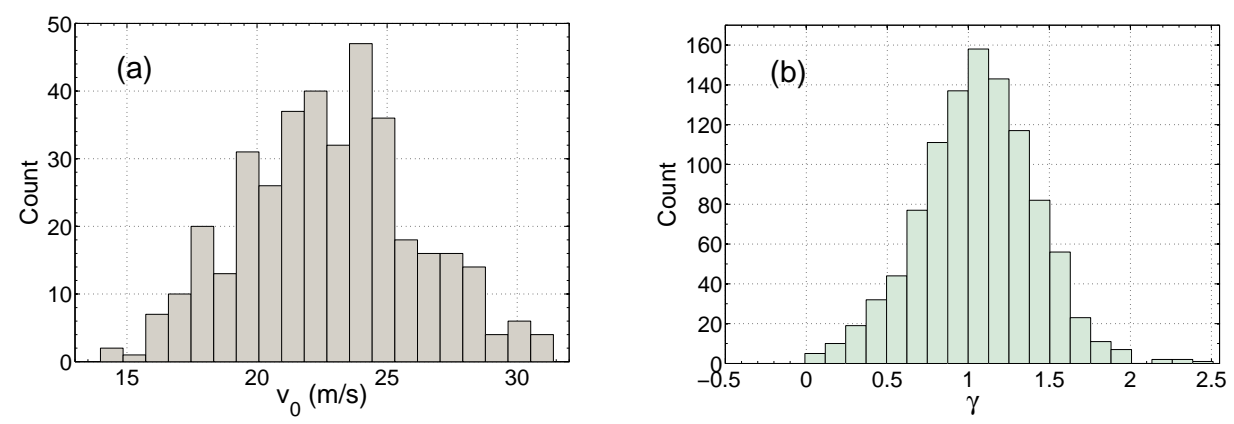

Fig. 1 Typical histograms of (a) the strength of the meridional circulation $v_{0}$ (left plot) and (b) the strength of the poloidal field $\gamma$ (right plot) used for grand minima simulations. These randomly generated data are taken from Gaussian distributions whose means and standard deviations are derived in CK12.
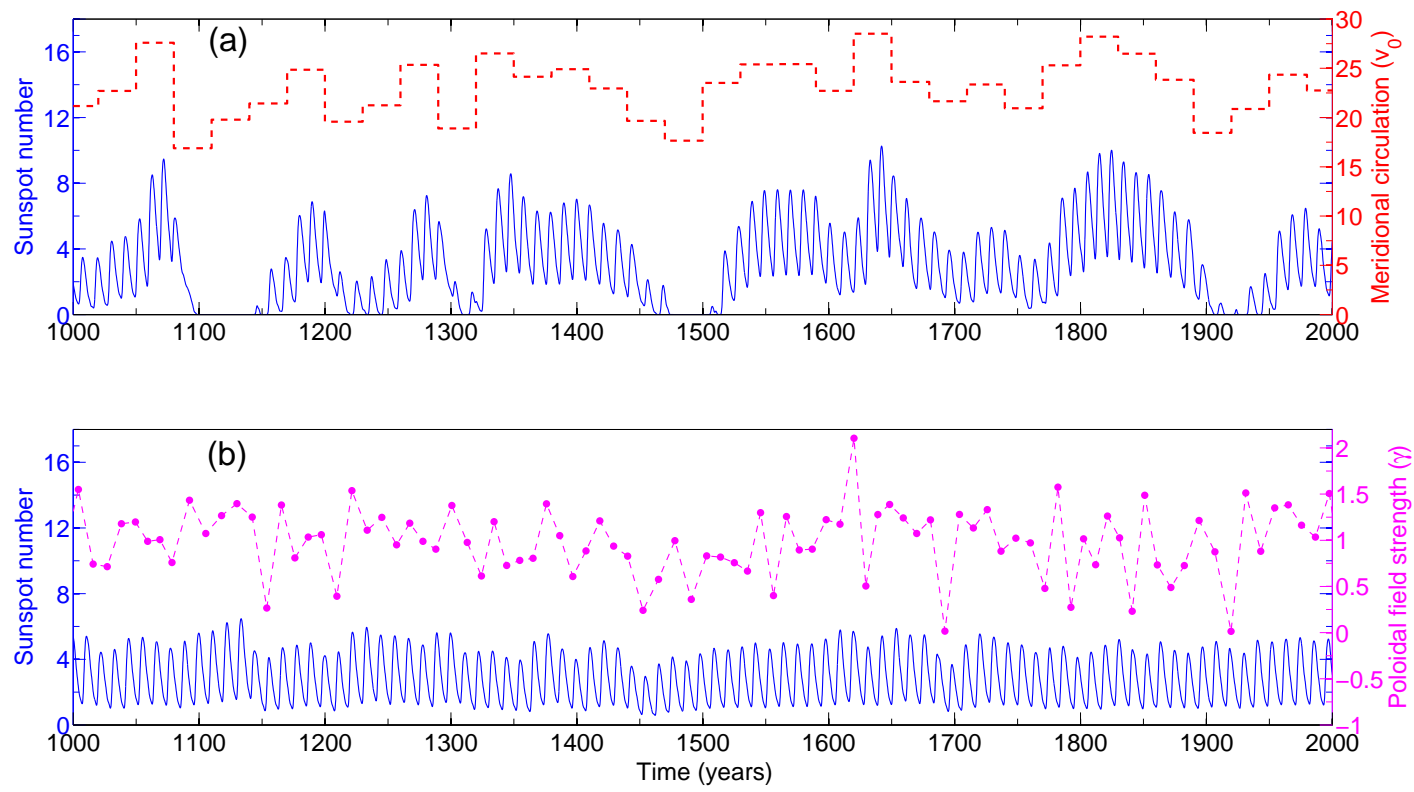

Fig. 2 (a) Upper panel: Simulation of grand minima with fluctuating meridional circulation with $\tau_{M C}=30$ years. The dashed (red) line shows the strength of meridional circulation $v_{0}$ used in the simulation whereas solid (blue) line shows the (theoretical) sunspot number as a function of time (years). Two grand minima are clearly evinced. (b) Bottom panel: Simulation with fluctuating poloidal field. The dashed (pink) line shows the strength of poloidal field $\gamma$ changed at every solar minimum, whereas solid (blue) line shows the sunspot number.

Based on these simulations, we make the following important conclusions.

i) The meridional circulation plays an important role in modulating the solar cycle period and the amplitude (consistent with Karak 2010). This is clear from Figure2 (a).

ii) Most of the grand minima are produced when the meridional circulation becomes sufficiently weak, 
els in Figure 2). Fluctuations in poloidal field has some effect in producing grand minima when combined with fluctuating meridional circulation, but are not able to produce any grand minima separately.

Although we ourselves had not been aware of these conclusions at the time of writing our earlier paper CK12, it now seems from hindsight that these conclusions would follow from Figure 1 of CK12, where the region of the parameter space required for producing grand minima is demarcated. When the polar field is not varied (i.e. $\gamma$ is always kept equal to 1 ), we see from Figure 1 of CK12 that it is necessary to make $v_{0}$ less than about $15.5 \mathrm{~m} \mathrm{~s}^{-1}$ to produce grand minima and we find that the probability of this is not too low, as can be seen from Figure 2(a) of CK12. On the other hand, if fluctuations in meridional circulation are not included (i.e. $v_{0}$ is held fixed at the value $23 \mathrm{~m} \mathrm{~s}^{-1}$ ), then $\gamma$ has to be made about -1 (below the bottom of Figure 1 of CK12) and we see from Figure 2(b) of CK12 that the probability of this is miniscule.

Now we come to the question whether our conclusion that the fluctuations in the polar field do not play an important role in producing grand minima changes when the parameters of the basic dynamo model are different. This is discussed in the next subsection.

\subsection{Sensitivity of the results on the value of $\alpha$}

When we run our basic dynamo model without fluctuations, we find that the critical value of the $\alpha$ coefficient is $\alpha_{0}=21.1 \mathrm{~m} \mathrm{~s}^{-1}$. In other words, when we run the code by varying $\alpha_{0}$ alone and keeping all the other parameters fixed (especially using the diffusivity $\eta_{0}=3 \times 10^{12} \mathrm{~cm}^{2} \mathrm{~s}^{-1}$ ), we get non-decaying oscillatory solution only if $\alpha_{0}$ is larger than this critical value. The results of CK12 as well as the results presented in Section 3.1 are based on a model using a somewhat supercritical value $\alpha_{0}=30 \mathrm{~m} \mathrm{~s}^{-1}$. We now carry on some calculations using only a moderately critical value $\alpha_{0}=24 \mathrm{~m} \mathrm{~s}^{-1}$. We study the effect of introducing fluctuations in polar field alone on this moderately critical dynamo. Figure 3 shows a typical result of such a simulation. Now we find that 14 grand minima are produced in 11,000 yr. It is not difficult to give a physical argument why fluctuations in polar field produce grand minima more easily in a moderately critical dynamo. If the fluctuations make the polar field during a minimum much less than the polar field which such a moderately critical dynamo would normally produce, then this is almost as if the strength of $\alpha$ is temporarily reduced and this can make the dynamo subcritical, pushing it into a grand minimum. This is not likely to happen when the dynamo is reasonably supercritical. We thus conclude that fluctuations in the polar field would have a significant effect on the dynamo only if it is moderately critical.

We have done some calculations introducing fluctuations in both the meridional circulation and the poloidal field in a moderately critical dynamo. The number of grand minima becomes much larger than the observed value. Since the results of a supercritical dynamo are in such good agreement with the observations (CK12), one is tempted to conclude that the solar dynamo is supercritical and fluctuations in the polar field do not have much effect in inducing grand minima. It may be noted that Charbonneau et al. (2007) proposed that the Gnevyshev-Ohl effect is produced by period doubling, which also requires a reasonably supercritical dynamo. Other dynamo models which are not supercritical show the occurrence of grand minima on introducing fluctuations in $\alpha$-effect alone (Olemskoy et al. 2013).

We point out that the earlier paper by Choudhuri \& Karak (2009) produced grand minima only with 


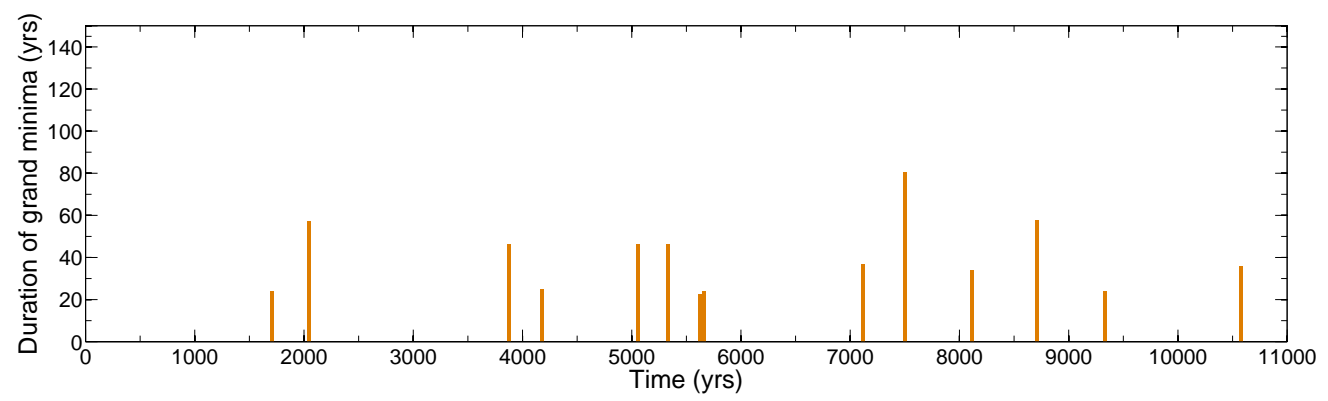

Fig. 3 The durations of grand minima indicated by vertical bars at their times of occurrence in a 11,000 yr simulation with only poloidal field fluctuations. Note that unlike earlier in this case we get 14 grand minima with poloidal field fluctuations alone. In this simulation $\alpha_{0}=24 \mathrm{~m} \mathrm{~s}^{-1}$ instead of $30 \mathrm{~m} \mathrm{~s}^{-1}$ used earlier; everything else remain unchanged.

Additionally, one ad hoc assumption used in this paper was to reduce the toroidal field also by a factor 0.8 when the poloidal field was reduced to create a grand minimum. This helped in creating the Maunder-like grand minima. In the present paper, we do not use this ad hoc assumption and the toroidal field is never changed when changing the poloidal field to incorporate fluctuations in the Babcock-Leighton process.

The results presented in the remaining subsections of this section are all obtained with the supercritical dynamo used in CK12.

\subsection{Are initiations of grand minima sudden or gradual?}

One important question connected with grand minima is whether they initiate suddenly or gradually. Usoskin et al. (2000) concluded that the Maunder minimum started abruptly. However, Vaquero et al. (2011) now present evidence that the initiation of the Maunder minimum was more gradual. On the theoretical side, in our simulation runs we find both grand minima which start abruptly and which start gradually. Since we now recognize the fluctuations in meridional circulation to be the primary cause of grand minima, we discuss the results for the run with fluctuations in meridional circulation alone. Things can be seen more cleanly there. The results are qualitatively the same when both fluctuations in meridional circulation and polar field are present.

In Figure 2 a) we see that the grand minimum that started slightly before 1500 and also the grand minimum that started around 1900 initiated gradually. These grand minima did not start immediately after the meridional circulation became sufficiently weak. The dynamo took about one or two solar cycles to enter into grand minima. Therefore, one or two solar cycles before the beginning of grand minima, the solar cycle period tends to become longer (because the meridional circulation determines the cycle period). This result is remarkably consistent with the results of Miyahara et al. (2010) who have found sufficient evidences of the longer solar cycles even before the beginning of the Maunder minimum and also the Spörer minimum.

Now we discuss an opposite case where the grand minimum starts suddenly and we do not see much change of the solar cycle period before the beginning of the grand minimum. In Figure 2 a), the grand minimum around 1100 shows this behaviour. We note that this grand minimum was caused by the meridional 
period did not get elongated before the start of the grand minimum, the cycle period is longer than the usual during the actual grand minimum epoch.

Based on our theoretical results, we draw the following conclusion. As the meridional circulation is made to fluctuate randomly, it would sometimes happen that the meridional circulation would drop from a rather high value to a low value. In such a situation, the initiation of the grand minimum seems abrupt. More commonly, we may have the meridional circulation dropping from a more moderate value to a low value. The grand minimum starts more gradually in this situation. As we do not know at present how rapidly the meridional circulation can drop to a low value, we do not know which one is more physical. In our simulations done with both kinds of fluctuations present, we have noted that there are about $40 \%$ grand minima which initiate abruptly whereas the remaining grand minima initiate more gradually. However the recovery from grand minima is always gradual, which is consistent with observations during the Maunder minimum (Usoskin et al. 2000).
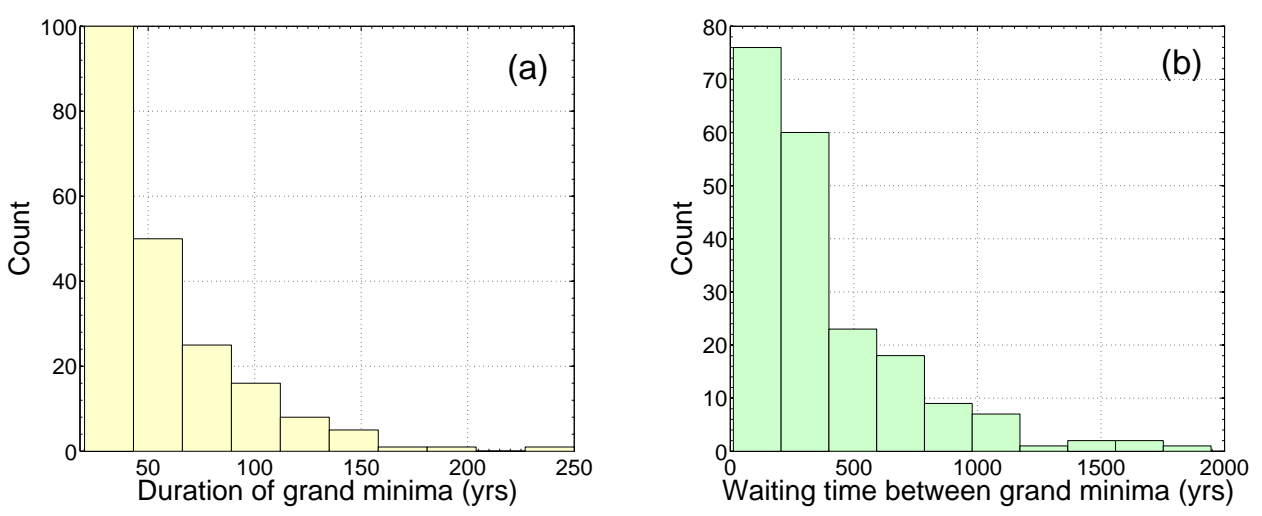

Fig. 4 Left panel (a) shows the distribution of the durations of the grand minima and the right panel (b) shows the distribution of the waiting times between the grand minima. This figure is produced from the data of 88,000 years of simulation run.

\subsection{Statistics of grand minima}

In Figure 5 of CK12 we presented the distributions of durations of grand minima and the waiting times between them. Since these distributions were constructed from the limited set of 29 grand minima which occurred during one run, the nature of these distributions was not very clear from this figure. To make a statistically reliable conclusion, we now make histograms from the data of a very long simulation (about 88,000 years) in which we have detected about 207 grand minima. The histograms are shown in Figure 4 From this plot, we now clearly see that both the duration and the waiting time follow exponential behaviour. This tells that the duration and waiting time are governed by stationary memoryless stochastic processes. As the grand minima are produced by the random fluctuations in the meridional circulation and the poloidal field, the occurrence of the grand minimum must be random events implying that the waiting time distribution is exponential. On the other hand, once the dynamo enters into a grand minimum state, the recovery of dynamo from grand minima state is only possible by the increase of meridional circulation which hap- 
dynamo into the normal cycle, the distribution of the durations of grand minima is also exponential. We mention that the observational distribution of the waiting times of grand minima based on 27 grand minima in last 11,400 years reported by Usoskin et al. (2007) is also exponential, whereas the distribution of durations is not so conclusive.

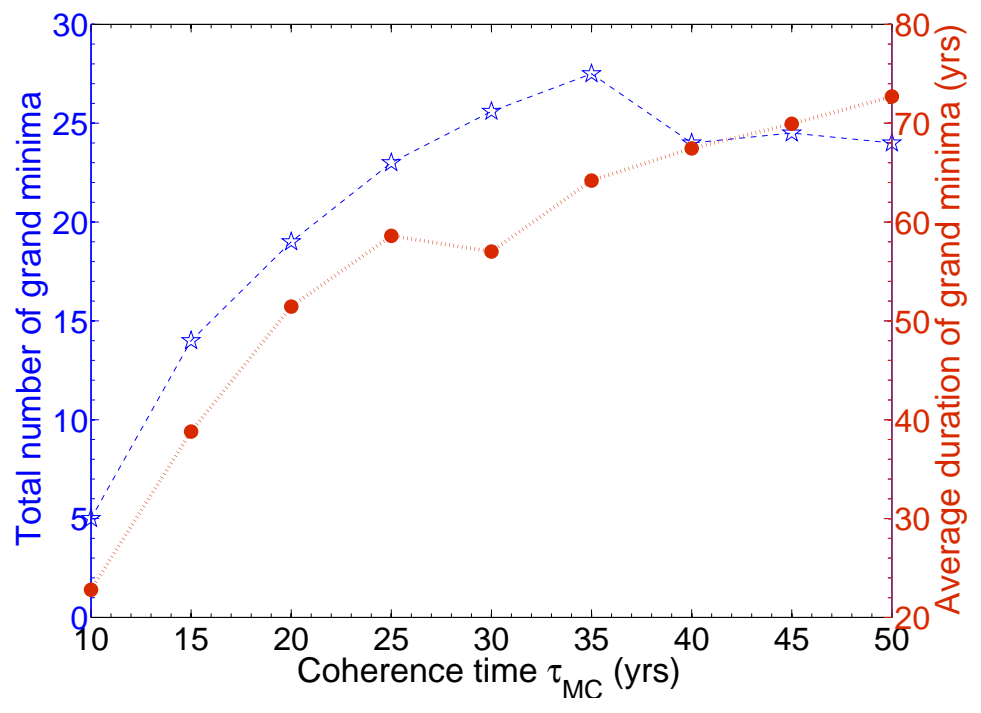

Fig. 5 Dependence of the number of grand minima (shown by star and dashed line) in 11,000 years and their average duration (shown by circle and dotted line) along right vertical axis as a function of the coherence time of the meridional circulation $\left(\tau_{\mathrm{MC}}\right)$.

\subsection{The dependence of the coherence time of meridional circulation}

Karak \& Choudhuri (2011) pointed out that several successive cycles in the past often had very similar periods (see their Figure 2). This suggests that the meridional circulation probably had remained steady during those cycles before changing abruptly at the end of such an epoch. Given the limited data of the last few cycles, it is very difficult to estimate the coherence time $\tau_{M C}$ of the meridional circulation. Karak \& Choudhuri (2011) concluded that this coherence time should lie in the range between 15 years and 45 years. All the results presented in CK12 were obtained by using a coherence time of 30 years. Here we explore the importance of this coherence time $\tau_{M C}$ of the meridional circulation on various features of grand minima.

In our earlier simulations of CK12 using $\tau_{M C}=30$ years, after every 30 years $v_{0}$ was varied randomly in accordance with their distributions. Now we have performed several simulations by varying $\tau_{M C}$ from 10 to 50 years. We note the number of grand minima in a run of 11,000 years and also calculate the average duration of such grand minima for each run with a particular value of $\tau_{M C}$. Note that in all simulations the poloidal field is changing at every solar minimum only. Figure 5 shows the results. We see that the total number of grand minima initially increases with the increase of $\tau_{M C}$ and then, after a certain value around 30 years, it tends to decrease. This is easy to understand. If $\tau_{M C}$ is small, the dynamo does not get much time to make magnetic fields sufficiently weak even when the meridional circulation falls to a 
the increase of $\tau_{M C}$, the number of grand minima increases. However, after $\tau_{M C}$ becomes comparable to the typical duration of a few cycles, further increase of it does not increase the number of grand minima rather it decreases. If the meridional circulation changes after long times, then the probability that it falls to a sufficiently low value also becomes less. Therefore the total number of grand minima in a finite period of run becomes less at large $\tau_{M C}$.

Another important result from this figure is that the average duration of grand minima is an increasing function of $\tau_{M C}$. In an earlier work with constant meridional circulation where grand minima were produced by fluctuations in polar field alone, Choudhuri \& Karak (2009) found that the recovery to the normal state from a grand minimum is only determined by the dynamo growth rate (measured by the dynamo number $\sim \alpha / \eta^{2}$; where $\alpha$ is the strength of the poloidal field generation process during grand minimum episode). However, in the case of fluctuating meridional circulation, where grand minima are mostly caused by the weak meridional circulation, the recovery from grand minima state is not only determined by the dynamo number but also on how rapidly the meridional circulation comes back to a more usual value from the very low value that caused the grand minimum. If the meridional circulation recovers quickly from its low value, the duration of the grand minimum will be short and vice versa. Therefore the average duration of grand minima is strongly dependent on how frequently the meridional circulation changes, i.e., on $\tau_{M C}$.

\subsection{Grand maxima}

Of late, grand maxima-epochs during which solar activity becomes exceptionally strong for sufficiently long time-are drawing more and more attention of solar physicists. The middle of the twentieth century was such an epoch when successive several solar cycles were rather strong (Solanki et al. 2004). Along with grand minima, Usoskin et al. (2007) also presented a study of grand maxima during the last 11,400 years. They identified 19 grand maxima and showed that their durations follow an exponential distribution, suggesting that the durations are determined by a memoryless random process.

We present a study of grand minima from our theoretical simulation. How you define grand maxima has more arbitrariness compared to how you define grand minima. We now explain how we select our grand maxima. Figure 6 a) plots the theoretical sunspot eruptions in the numerical run, whereas Figure 6b) is a histogram showing the distribution of the peak values of these cycles. The solid horizontal line in Figure 6a) and the solid vertical line in Figure 6 b) indicate the mean value $S N_{m}$ of the cycle peaks. We calculate the standard deviation $(\sigma)$ of these peak values. The dashed (red) lines in Figure 6 a) and Figure 6 (b) indicate the $S N_{m}+\sigma$ levels. If at least two successive solar cycles have their strengths above this level, then we take it as a grand maximum. Figure 6 (c) and Figure 6 d) respectively are the histograms showing the distribution of the durations and the waiting times of these grand maxima. To figure out the extent to which the statistical distributions depend on the definition of grand maxima, we also present results by defining grand maxima as at least two successive cycles having peaks above $S N_{m}+1.2 \sigma$ levels. These levels are indicated in Figure 6a) and Figure 6 b) by the dash-dotted (pink) lines. The distributions of durations and waiting times for grand maxima defined in this way are shown in Figure 6 (e) and Figure 6 f). Comparing them with Figure 6 (c) and Figure 6(d), we conclude that the statistical behaviours of grand maxima are reasonably 

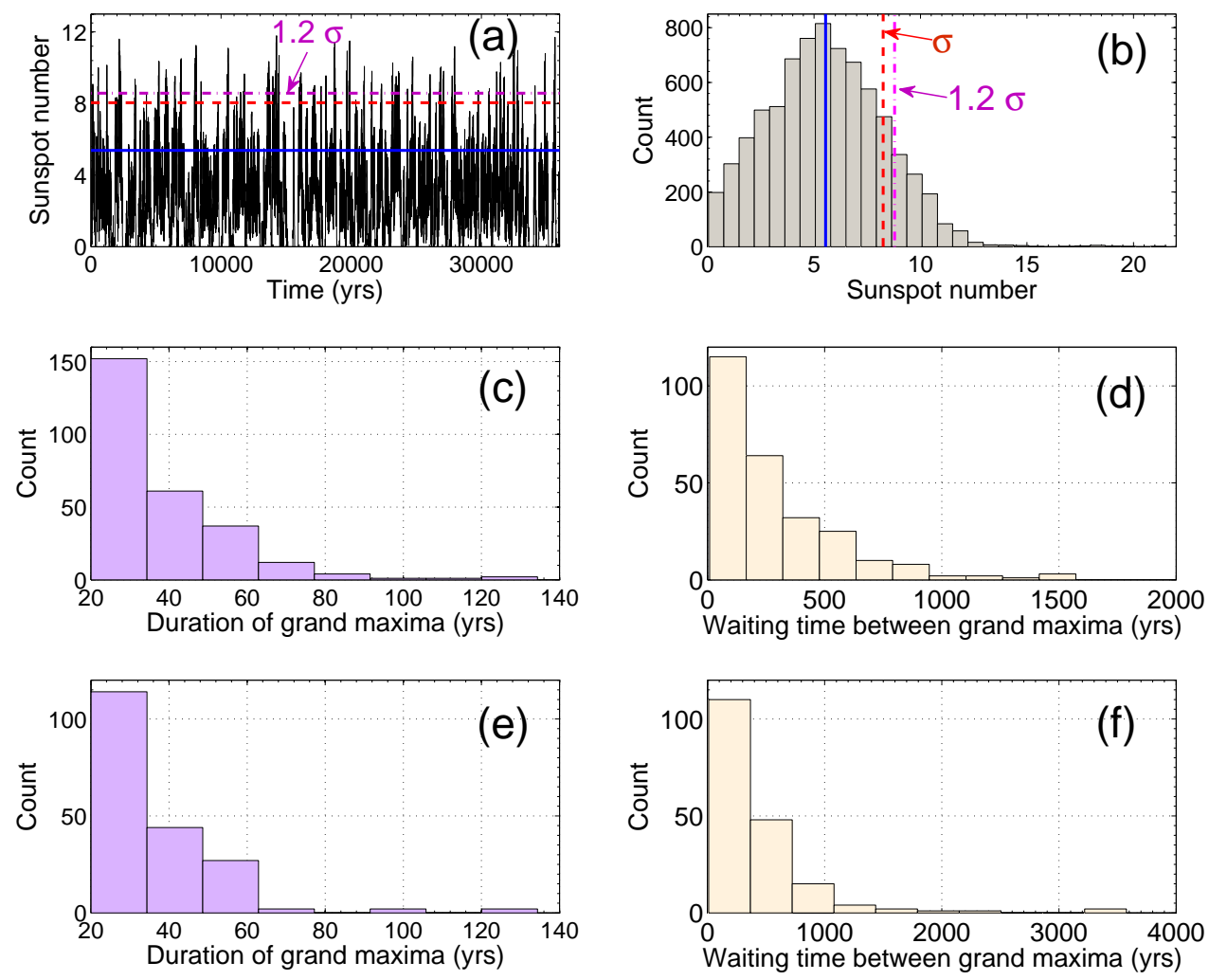

Fig. 6 Statistics of grand maxima. (a) Shows the theoretical sunspot number. The three horizontal lines (solid, dashed and dash-dotted) indicate the values $S N_{m}, S N_{m}+\sigma$ and $S N_{m}+1.2 \sigma$, where $S N_{m}$ and $\sigma$ are the mean and the standard deviation of the peak sunspot numbers. (b) Shows the histogram of the peak sunspot numbers where the three vertical lines (solid, dashed and dashdotted) indicate the values $S N_{m}, S N_{m}+\sigma$ and $S N_{m}+1.2 \sigma$. (c) and (d) Show the histograms of durations and waiting times of grand maxima defined to be above the $S N_{m}+\sigma$ level. (e) and (f) Show histograms similar to (c) and (d), except that the grand maxima are now defined to be above the $S N_{m}+1.2 \sigma$ level.

We remind the reader that, to get a statistically significant result, we used data of about 88,000 years long run. The numbers of grand maxima were 270 and 191 in the two definitions. This implies that the numbers of grand maxima in 11,000 years would be 34 and 24. Interestingly the distributions of durations and the waiting times of grand maxima as shown in Figure6(c)-(d) also seem to follow the exponential distribution, which again tells us that the occurrence of grand maxima is governed by a stationary memoryless random process. On comparing with Figures 4 (a)-(b) showing similar distributions for grand minima, we note that the durations of grand maxima tend to be shorter than the durations of grand minima. A physical explanation for this is not difficult to give. Once the Sun enters a grand minimum due to an effect like the slowing down of the meridional circulation, the dynamo has to build up the magnetic field again before the Sun can come out of the grand minimum even after the meridional circulation has returned to more normal values. This 
easily when conditions change due to stochastic fluctuations. Another important point to note is that like grand minima grand maxima are mainly caused by the variable meridional circulation. This becomes clear by looking at Figure 2 a). We see that strong meridional circulation makes the cycle stronger.

\section{THE RECOVERY PHASE OF GRAND MINIMA}

Here we explore an important, yet unsettled, issue connected with grand minima: the recovery mechanism from grand minima states. If the meridional circulation or the poloidal field somehow becomes sufficiently weak, then that can push the Sun into a grand minimum. However, we do not understand well how the Sun comes out of such a quiescent state. There are also many uncertainties in our understanding of the nature of the dynamo process during the grand minimum state. It has been clearly demonstrated by observations that the solar cycle continued during grand minima with weaker strength and also with polarity reversals (Fligge et al. 1999; Miyahara et al. 2004, 2010; Nagaya et al. 2012). The question that remains open is how the poloidal field is generated during grand minima. The Babcock-Leighton process depends on the decay of tilted active regions and at present we have strong observational evidence that this process is indeed working near the solar surface (Dasi-Espuig et al. 2010; Kitchatinov \& Olemskoy 2011; Jiang et al. 2013). On the other hand, very few sunspots were detected during the Maunder minimum (Sokoloff \& NesmeRibes 1994; Hoyt \& Schatten 1996). Therefore, the Babcock-Leighton process may have been ineffective during the Maunder minimum. However, in all our earlier calculations (Choudhuri \& Karak 2009; Karak 2010; CK12), we have used the same $\alpha$ concentrated near the solar surface corresponding to the BabcockLeighton mechanism all the time for the poloidal field generation because of our lack of knowledge about handling the problem in a better way. Now we assume that the Babcock-Leighton process cannot operate when the toroidal field is very weak and sunspots do not form. We make the Babcock-Leighton $\alpha$ coefficient fall to zero when the dynamo enters a grand minimum and keep running the simulation. Then the dynamo cannot come out of the grand minimum state, as seen in Figure 7

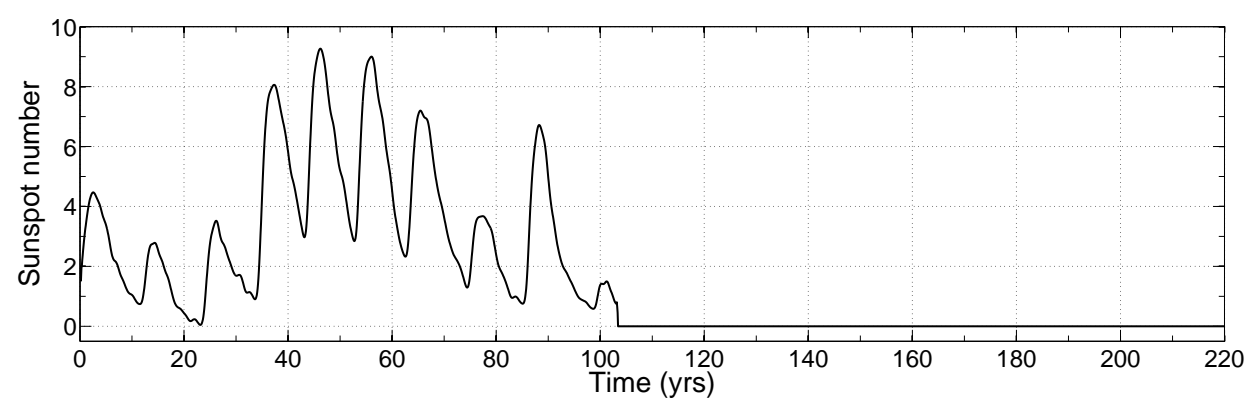

Fig. 7 Simulation of the solar cycle with the Babcock-Leighton mechanism, which gets switched off when there are no sunspots. The dynamo is not able to recover from the grand minimum state.

If we assume that the Babcock-Leighton process cannot work during a grand minimum, we need some other mechanism to pull the dynamo out of the grand minimum. The obvious other candidate to produce the poloidal field is the $\alpha$-effect based on helical turbulence $\left(\alpha_{\mathrm{HT}}\right)$ proposed by Parker (1955) and Steenbeck et al. (1966). This is a mechanism of generating the poloidal field in the convection zone by the twist of 
that sunspots form from toroidal magnetic fields as strong as $10^{5} \mathrm{G}$ (Choudhuri \& Gilman 1987; Choudhuri 1989; D'Silva \& Choudhuri 1993; Fan et al. 1993), the Babcock-Leighton mechanism was favoured over the $\alpha$-effect in the flux transport dynamo model, since the helical turbulence cannot twist toroidal fields as strong as $10^{5} \mathrm{G}$. Presumably the situation gets reversed during a grand minimum. While the BabcockLeighton mechanism may not be operative due to the lack of sunspots, the weaker toroidal magnetic field during the grand minimum may allow the $\alpha$-effect to work. We now present some simulations in which we switch on an $\alpha$-effect during the grand minima and investigate what conclusion we can draw about the nature of the $\alpha$-effect by requiring that results of the simulation agree with observations. These simulations pertaining to the plausible failure of the Babcock-Leighton dynamo to recover from a grand minimum episode and the role of an additional mean-field alpha effect in a possible recovery are motivated by discussions with Dibyendu Nandy and Soumitra Hazra (private communications) and the work detailed in Passos (2010, PhD Thesis), Hazra, Passos and Nandy (2013) and Passos, Hazra and Nandy (2013). One other point needs to be noted. Because of the way we treat magnetic buoyancy in our code, whenever the toroidal field strength above the bottom of the convection zone exceeds $B_{c}$, a part of it is brought to the solar surface. During the usual situation (i.e. outside grand minima), the toroidal field near the surface in our simulation continuously gets enhanced by magnetic buoyancy. This does not happen during the grand minima. Whether we allow the Babcock-Leighton process to continue or replace it by the $\alpha$-effect, the toroidal field at the solar surface during grand minima comes there due to turbulent diffusion or advection due to the meridional circulation after being created in the tachocline.

As soon as the dynamo enters into a grand minimum state and the sunspot eruption stops due to the weak toroidal field, we switch off the $\alpha_{\mathrm{BL}}$ corresponding to the Babcock-Leighton mechanism and switch on the $\alpha_{\mathrm{HT}}$ representing the twisting of the toroidal field by helical turbulence. Then, after the recovery from the grand minimum state, we switch off the $\alpha_{\mathrm{HT}}$ and again switch on the $\alpha_{\mathrm{BL}}$. Although the nature and also the sign of this $\alpha_{\mathrm{HT}}$ is not certain at present, we use the following profile for it:

$$
\alpha_{\mathrm{HT}}=1.1 \cos \theta \frac{1}{2}\left[1+\operatorname{erf}\left(\frac{r-0.85 R_{\odot}}{0.025 R_{\odot}}\right)\right] \mathrm{m} \mathrm{s}^{-1}
$$

The profile of this $\alpha_{\mathrm{HT}}$ along with the Babcock-Leighton $\alpha_{\mathrm{BL}}$ is shown in Figure 8 Note that $\alpha_{\mathrm{HT}}$ is almost one order of magnitude smaller than the $\alpha_{\mathrm{BL}}$ and importantly $\alpha_{\mathrm{HT}}$ is zero below around $0.8 R_{\odot}$. We have seen that, if $\alpha_{\mathrm{HT}}$ is non-zero within the whole body of the convection zone, then the solar cycle periods during grand minima become very short, which is not supported by the observation (Fligge et al. 1999; Miyahara et al. 2004, 2010; Nagaya et al. 2012). One important conclusion we draw is that an $\alpha$-effect which gets switched on during grand minima has to be restricted in the upper regions of the convection zone if we do not want the periods to become too short.

Now let us comment on the amplitude of $\alpha_{\mathrm{HT}}$. If $\alpha_{\mathrm{HT}}$ has the amplitude $1.1 \mathrm{~m} \mathrm{~s}^{-1}$ in the upper part of the convection zone which follows from (3), the results of the simulation are qualitatively exactly similar to the results we got by allowing the Babcock-Leighton $\alpha_{\mathrm{BL}}$ to operate all the time. The results of the simulations of the grand minima with this $\alpha_{\mathrm{HT}}$ during grand minima is shown in Figure 9 . This plot shows the positions of the grand minima along the time axis whereas the vertical axis shows the durations of grand minima. In this 11,000 years simulation run, we get about 28 grand minima. A comparison with Figure 3 of 


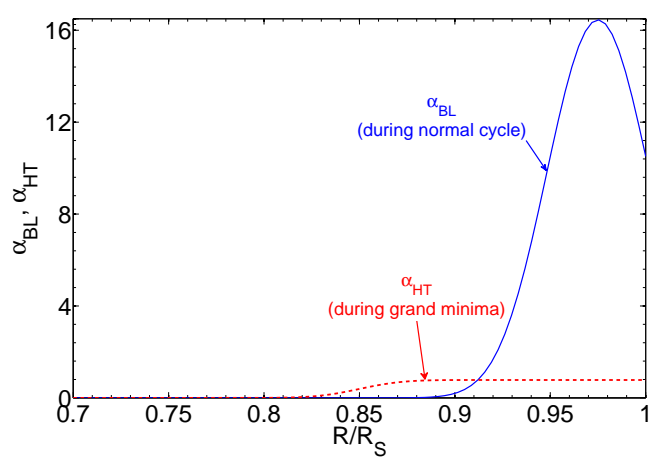

Fig. 8 Variations of the strength of the Babcock-Leighton $\alpha_{\mathrm{BL}}$ (solid line) and the (helical) turbulent $\alpha_{\mathrm{HT}}$ (dashed line) as a function of solar radius at $45^{0}$ latitude.

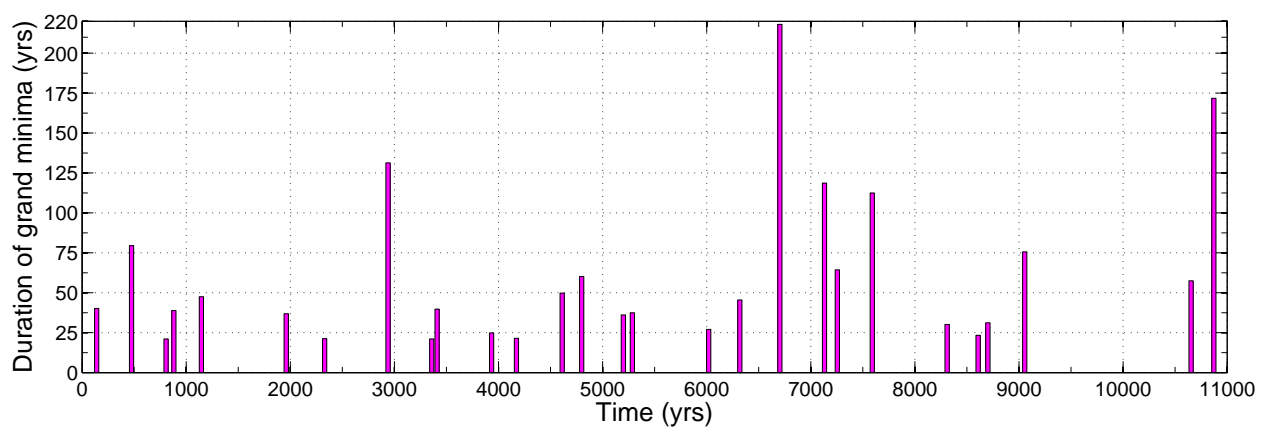

Fig. 9 The durations of grand minima indicated by vertical bars at their times of occurrence in a $11,000 \mathrm{yr}$ simulation. This is the result of a particular realization of random fluctuations that produced 28 grand minima. In this simulation, during grand minima episodes, the BabcockLeighton $\alpha_{\mathrm{BL}}$ is switched off and a weak turbulent $\alpha_{\mathrm{HT}}$ is allowed to switch on with the amplitude $1.1 \mathrm{~cm} \mathrm{~s}^{-1}$.

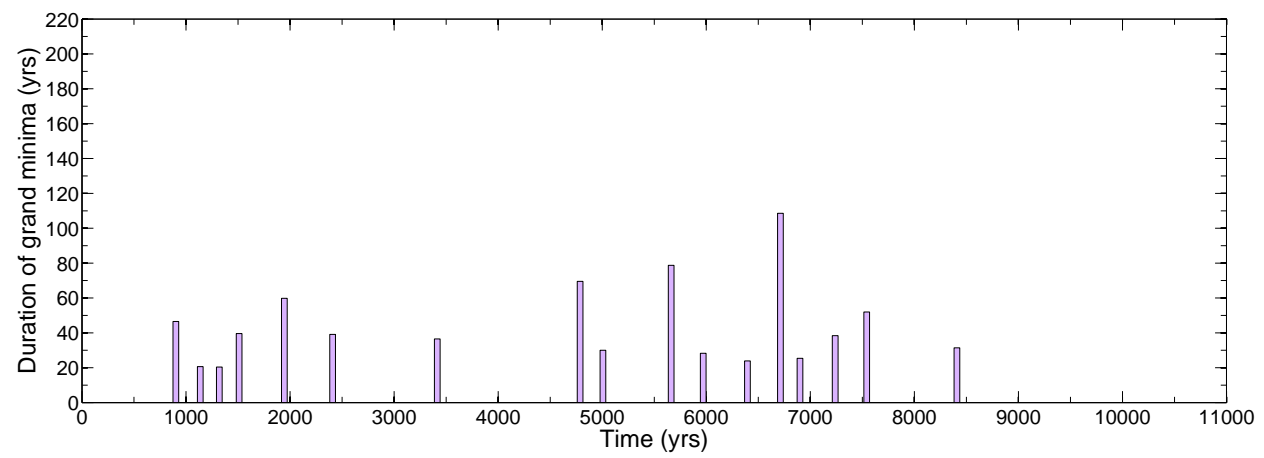

Fig. 10 The same as Figure 9 except that turbulent $\alpha$ switched on during the grand minima has the amplitude $1.2 \mathrm{~cm} \mathrm{~s}^{-1}$.

to have the slightly larger amplitude $1.2 \mathrm{~m} \mathrm{~s}^{-1}$. We see that the number of grand minima is reduced in this situations. When $\alpha_{\mathrm{HT}}$ is larger, the dynamo gets out of the state of reduced activity very quickly. Since we 

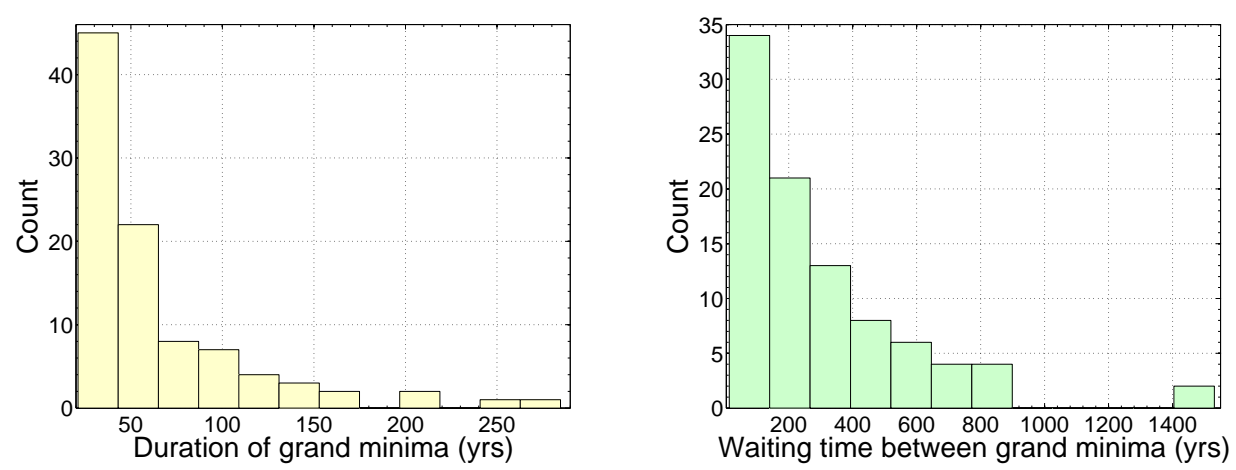

Fig. 11 Same as Figure 4 except that the turbulent $\alpha_{\mathrm{HT}}$ gets switched on during grand minima instead of Babcock-Leighton $\alpha_{\mathrm{BL}}$ operating all the time.

reduced. We also did runs by using the slightly lower value of the amplitude $1.0 \mathrm{~m} \mathrm{~s}^{-1}$ for $\alpha_{\mathrm{HT}}$. In this case, we found that the dynamo was unable to get out of a grand minimum after entering it. Figure 11 presents histograms of the durations and the waiting times of grand minima from 33,000 years long simulation data using the value of $\alpha_{\mathrm{HT}}$ given by (3), with amplitude $1.1 \mathrm{~m} \mathrm{~s}^{-1}$. Again in this case both the distributions are exponential and qualitatively similar to the distributions shown in Figure 4

We are not completely sure what conclusions we should make out of the results we have presented. It is quite remarkable that the results of our simulation by using the same Babcock-Leighton $\alpha$ all the time, as had been done in CK12 and in Section 3 of this paper, are in such good agreement with different aspects of observational data. On the other hand, if we switch off this Babcock-Leighton $\alpha$ concentrated near the solar surface during the grand minima and use the traditional $\alpha$-effect to pull the dynamo out of the grand minima, then we have to fine-tune the nature of this $\alpha_{\mathrm{HT}}$ quite a bit in order to get results consistent with observational data. Interestingly, we get the best results when the amplitude of the $\alpha$-effect is just marginally above the critical value and only operating in the upper half of the convection zone. Does this tell us that Babcock-Leighton $\alpha$ remains operative even during the grand minima for reasons we do not understand now? This is a very provocative question which needs further investigation. Smaller active regions with magnetic flux less than that of detectable sunspots may have some (statistical) systematic tilt to produce a significant poloidal field during grand minima-like episodes (see the discussion in Wang \& Sheeley 2013). In fact, Stenflo \& Kosovichev (2012) find a systematic tilt for a long range of the magnetic fluxes in active regions, suggesting that the poloidal field may be generated when there are no detectable sunspots. Importantly, even a few big sunspots (with correct tilt) can produce a significant poloidal field to maintain the polarity reversal-this might also be the case during grand minima. As we do not have sufficient observational study of all these issues during the Maunder minimum, we cannot conclusively say anything about the poloidal field generation mechanism during grand minima. However Passos (2010, PhD Thesis), Passos et al. (2013) and Hazra et al. (2013) believe the Babcock-Leighton process cannot operate during the grand minimum episode and a weak mean-field $\alpha$ in the whole convection zone is needed to recover the Sun out of such phase based on their simulations with flux transport dynamo model and low 


\section{CONCLUSION}

The aim of the present paper is to follow up our earlier paper CK12 in exploring whether different aspects of grand minima can be explained on the basis of the flux transport dynamo model of the solar cycle. Following earlier work done by our group, we keep using a reasonably high turbulent diffusivity, which implies that a slowing down of the meridional circulation results in longer and weaker cycles. In such a model, the meridional circulation plays a profound role in producing irregularities of the solar cycle and also the grand minima. One of the main uncertainties in theoretical models at the present time is our lack of understanding of the meridional circulation, either from the theoretical or the observational viewpoint. While we do not yet have a complete theory of the solar meridional circulation, we believe that the turbulent stresses in the solar convection zone drives it and hence we assume the meridional circulation to be confined within the solar convection zone. The poleward meridional circulation near the solar surface causes the advection of the poloidal field to higher latitudes (Wang et al. 1989; Dikpati \& Choudhuri 1994, 1995). We need an equatorward counterflow at the bottom of the convection zone for flux transport dynamo to produce proper butterfly diagrams (Nandy \& Choudhuri 2002). So far we do not have direct observational measurements of this counterflow. While helioseismology has been able to provide information about meridional circulation in the upper layers of the convection zone (Giles et al. 1997; Braun \& Fan 1998), extracting unambiguous information about meridional circulation in the deeper layers has remained a challenge (Gough \& Hindman 2010; Zhao et al. 2012). Any possible periodic modulations of the meridional circulation with the solar cycle is not expected to produced sustained irregularities of the cycle (Karak \& Choudhuri 2012; Passos \& Lopes 2012). But random fluctuations of the meridional circulation with coherence times longer than solar cycle periods, as suggested by the data of past cycles, can have profound effects on the dynamo.

Our earlier paper CK12 suggested that grand minima are produced by combined fluctuations in the meridional circulation and in the Babcock-Leighton mechanism for generating the poloidal field. While our further calculations support this broad scenario, we now find that the fluctuations in the meridional circulation are more important in producing the grand minima (cf. Section 3.1). From the theoretical viewpoint, such variations of meridional circulationare not surprising. We know that the meridional circulation is mainly generated from the imbalance between two large terms - the non-conservative part of the centrifugal force and the baroclinic torque (i.e., the deviation from the thermal wind balance) (Kitchatinov and Rüdiger 1995). It is not only the case that there is a deviation which produces the meridional circulation in the solar convection zone but also this deviation fluctuates because of the fact that the differential rotation is produced by turbulent convection and the fluctuations in it is unavoidable (Brun et al. 2010). This physics already been explored by a mean-field model of Rempel (2005). He introduced random fluctuations in the $\Lambda$-effect and found that it produced fluctuations in the differential rotation but in turn the fluctuations produced in meridional circulation is about two orders of magnitude larger than that in the differential rotation. Indeed helioseismology has detected a significant temporal variation of the meridional circulation in last several years (e.g., González-Hernández et al. 2006). Unfortunately we do not have any measurement of the meridional circulation during the Maunder minimum. There are some observational studies which indicate 
suggesting also the variation of the meridional circulation. Some authors (Wang \& Sheeley 2003; Passos \& Lopes 2011) suggest weak meridional circulation during Maunder minimum.

Our calculations suggest that the fluctuations in the meridional circulation are more important than fluctuations in the polar field in inducing grand minima (cf. Section 3.1), although fluctuations in the polar field have more effect if the dynamo is only moderately critical (cf. Section 3.2). We have seen that the recovery phase is always gradual and supported by the observation. However, depending on the detailed nature of the fluctuations in the meridional circulation at the beginning of a grand minimum, we find that both sudden and gradual initiations of grand minima are possible. Since we are able to make only a very rough estimate of the coherence time of meridional circulation fluctuations, we explore how our results may change on varying the coherence time (cf. Section 3.5). For coherence times lying in the range 20-50 yr, the results remain qualitatively similar. We also present statistical analyses of the characteristics of grand minima (cf. Section 3.4). We have seen that both the distributions of the waiting times and the durations of the grand minima are exponential, suggesting that these are governed by the random process. Some of these results are supported by observational data (Usoskin et al. 2007). One issue we did not study here is the north-south asymmetry during grand minima. There are sufficient evidences that during the Maunder minimum and the Dalton minimum (Ribes \& Nesme-Ribes 1993; Usoskin et al. 2009b) there was strong north-south asymmetry in sunspots, indicating this to be a robust feature of grand minima. Choudhuri \& Karak (2009) and Karak (2010) have proposed that if the poloidal field or the meridional circulation becomes weak due to the stochastic fluctuations, then it is very unlikely that they become weak in both the hemisphere by the same amount. With this assumption they have demonstrated that by introducing a slight asymmetry in the poloidal field or in the meridional circulation we can easily model the observed north-south asymmetry of sunspots during Maunder minimum. We believe that the hemispheric asymmetry in grand minima may be another indication for the stochastic forcing as the origin of grand minima.

In this paper we have studied another interesting aspect of solar cycle which is grand maxima (cf. Section 3.6). We have seen that similar to grand minima, grand maxima are mostly caused by the strong meridional circulation and the distributions of both the waiting times and the durations of the grand maxima are exponential. Although the definition of grand maxima is more subjective, we have seen that the average duration of grand maxima are shorter compare to that of grand minima.

One other issue we addressed here is how the Sun comes out of a grand minimum. The BabcockLeighton mechanism for the poloidal field generation depends on the existence of sunspots and one naively thinks that this mechanism would not be operational during the grand minima. We explored whether the $\alpha$-effect, which gets suppressed when the toroidal field is strong, could be operational during the grand minima when the toroidal field becomes weak and whether this $\alpha$-effect could pull the Sun out of a grand minimum (cf. Section 4). We found that we can match various aspects of observational data only when we reside this $\alpha$-effect in the upper half of the convection zone and fine-tune its strength. On the other hand, on assuming that the Babcock-Leighton mechanism remains operational throughout the grand minima, we get results remarkably close to the observational data. This raises the provocative question whether the 
understand. We merely pose this question which cannot be answered at our present level of understanding of the subject.

Acknowledgements We thank the referee for careful reading and raising a few comments which improved the manuscript. We also thank Dibyendu Nandy and Soumitra Hazra for the discussion which motivated us to study the recovery phase of grand minima presented in Section 4. ARC thanks DST for partial support through JC Bose Fellowship (project no. SR/S2/JCB-61/2009). BBK thanks Nordita for providing the hospitality where the revised version of the article was prepared.

\section{References}

Babcock, H. W. 1961, ApJ, 133, 572

Brandenburg, A., \& Spiegel, E. A. 2008, Astron. Nachr, 329, 351

Braun, D. C., \& Fan, Y. 1998, ApJ, 508, L105

Brun, A. S, Antia, H. M., \& Chitre, S. M. 2010, A\&A 510, 33

Casas, R., Vaquero, J. M. \& Vazquez, M. 2006, Sol. Phys., 234, 379

Charbonneau, P., Blais-Laurier, G., \& St-Jean, C. 2004, ApJ, 616, L183

Charbonneau, P., Beaubien, G., \& St-Jean, C. 2007, ApJ, 658, 657

Charbonneau, P. 2010, Liv. Rev. Sol. Phys., 7, 3

Chatterjee, P., \& Choudhuri, A. R. 2006, Sol. Phys., 239, 29

Chatterjee, P., Nandy, D., \& Choudhuri, A. R. 2004, A\&A, 427, 1019

Choudhuri, A. R. 1989, Sol. Phys. 123, 217

Choudhuri, A. R. 1990, A\&A, 239, 335

Choudhuri, A. R. 1992, A\&A, 253, 277

Choudhuri, A. R. 2011, Pramana, 77, 77

Choudhuri, A. R., Chatterjee, P., \& Jiang, J. 2007, Phys. Rev. Lett., 98, 1103

Choudhuri, A. R., \& Gilman, P. A. 1987, ApJ, 316, 788

Choudhuri, A. R., \& Karak, B. B. 2009, RAA, 9, 953

Choudhuri, A. R., \& Karak, B. B. 2012, Phys. Rev. Lett., 109, 171103 (CK12)

Choudhuri, A. R., Schüssler, M., \& Dikpati, M. 1995, A\&A, 303, L29

Dasi-Espuig, M., Solanki, S. K., Krivova, N. A., Cameron, R. \& Peñuela, T. 2010, A\&A, 518, 7

Dikpati, M., \& Charbonneau, P. 1999, ApJ, 518, 508

Dikpati, M., \& Choudhuri, A. R. 1994, A\&A, 291, 575

Dikpati, M., \& Choudhuri, A. R. 1995, Sol. Phys., 161, 9

D'Silva, S., \& Choudhuri, A. R. 1993, A\&A, 272, 621

Durney, B. R. 1995, Sol. Phys., 160, 213

Eddy, J. A. 1976, Science, 192, 1189

Fan, Y., Fisher, G. H., \& Deluca, E. E. 1993, ApJ, 405, 390

Fligge, M., Solanki, S. K. \& Beer, J. 1999 A\&A, 346, 313

Giles, P. M., Duvall, T. L., Scherrer, P. H., \& Bogart, R. S. 1997, Nature, 390, 52

Goel, A., \& Choudhuri, A. R. 2009, RAA, 9, 115

Gómez, D. O., \& Mininni, P. D. 2006, Adv. in Space Res., 38, 856

Gough, D., \& Hindman, B. W. 2010, ApJ, 714, 960

González-Hernández, I., Komm, R., Hill, F., Howe, R., Corbard, T., \& Haber, D. A. 2006, ApJ, 638, 576

Hazra, S., Passos, D. \& Nandy, D. 2013, in preparation

Hotta, H., \& Yokoyama, T. 2010, ApJ, 714, L308

Hoyng, P. 1988, ApJ, 332, 857

Hoyt, D. V., \& Schatten, K. H., 1996, Sol. Phys., 165, 181

Jiang, J., Chatterjee, P., \& Choudhuri, A. R. 2007, MNRAS, 381, 1527

Jiang, J., Cameron, R. H., Schmitt, D. \& Isik, E. 2013, A\&A, 553, 128

Karak, B. B. 2010, ApJ, 724, 1021

Karak, B. B., \& Choudhuri, A. R. 2011, MNRAS, 410, 1503 
Karak, B. B., \& Nandy, D. 2012, ApJ, 761, L13

Karak B. B. \& Choudhuri, A. R. 2013 IAU 294:Sol. and Astrophys. Dynamos and Magnetic Activity (arXiv:1211.0165)

Karak, B. B., \& Petrovay, K. 2013, Sol. Phys., 282, 321.

Kitchatinov, L. L., \& Rüdiger, G. 1995, A\&A, 299, 446

Kitchatinov, L. L., \& Olemskoy, S. V. 2011, Astron. Lett., 37, 656

Leighton, R. B. 1969, ApJ, 156, 1

Longcope, D., \& Choudhuri, A. R. 2002, Sol. Phys., 205, 63

Miesch, M. S, Featherstone, N. A., Rempel, M. \& Trampedach, R. 2012, ApJ, 757, 128

Miyahara, H., Masuda, K., Kyohei, K., Kentaro, N., Yusuke, Y., Hiroyuki, M., Kimiaki, M., Toshio, N., \& Yasushi, M. 2010, Journal of Cosmology, 8, 1970

Miyahara, H., Masuda, K., Muraki, Y., Furuzawa, H., Menjo, H., \& Nakamura, T. 2004, Sol. Phys., 224, 317

Miyahara, H., Masuda, K. Muraki, Y. Kitagawa, H. \& Nakamura, T. 2006, JGR, 111, A03103, doi:10.1029/2005JA011016

Miyahara, H., Masuda, K., Nagaya, K., Kuwana, K., Muraki, Y., \& Nakamura, T. 2007, Adv. Space Res., 40, 1060

Moss, D., Sokoloff, D., Usoskin, I. G., \& Tutubalin, V. 2008, Sol. Phys., 250, 221

Muñoz-Jaramillo, A., Dasi-Espuig, M., Balmaceda, L. A. \& DeLuca, E. E. 2013, ApJ, 767, L25

Nagaya, K., Kitazawa, K. Miyake, F., Masuda, K. Muraki, Y., Nakamura, T., Miyahara, H., \& Matsuzaki, H. 2012 , Sol.

Phys., 280, 223

Nandy, D., \& Choudhuri, A. R. 2002, Science, 296, 1671

Olemskoy, S. V., Choudhuri, A. R., \& Kitchatinov, L. L. 2013 Astronomy Reports, 57, 458

Parker, E. N. 1955, ApJ, 122, 293

Passos, D., \& Lopes, I. 2008, ApJ, 686, 1420

Passos, D., \& Lopes, I. P. 2011, JASTP, 73, 191.

Passos, D., \& Lopes, I. P. 2012, MNRAS, 422, 1709.

Passos, D., Charbonneau, P. \& Beaudoin, P. 2012, Sol. Phys. 279, 1

Passos, D., Hazra, S. \& Nandy, D. 2013, in preparation

Passos 2010, PhD Thesis, INSTITUTO SUPERIOR TÉCNICO

Rempel, M. 2005, ApJ, 631, 1286

Ribes, J. C., \& Nesme-Ribes, E. 1993, A\&A, 276, 549

Sokoloff, D., \& Nesme-Ribes, E. 1994, A\&A, 288, 293

Solanki, S. K., Usoskin, I. G., Kromer, B., Schussler, M. \& Beer, J., 2004, Nature, 431, 28

Spruit, H. C. 1981, A\&A, 98, 155

Steenbeck, M., Krause, F., \& Rädler, K.-H. 1966, Z. Naturforsch., 21a, 1285

Steinhilber, F., Abreu, J. A., Beer, J., et al. 2012, PNAS, 109, 5967

Stenflo, J. O., \& Kosovichev, A. G. 2012, ApJ, 745, 129

Usoskin, I. G., Mursula, K., \& Kovaltsov, G. A. 2000, A\&A, 354, L33

Usoskin, I. G., Sokoloff, D., \& Moss, D. 2009a, Sol. Phys., 254, 345

Usoskin, I. G., Mursula, K., Arlt, R., \& Kovaltsov, G. A. 2009b, ApJL, 700, L154

Usoskin, I. G., Solanki, S. K., \& Kovaltsov, G. A. 2007, A\&A, 471, 301

Vaquero, J. M., Gallego, M. C., Usoskin, I. G., \& Kovaltsov, G. A. 2011, ApJ, 731, L24

Wang, Y.-M., Nash, A. G., \& Sheeley, N. R. 1989, ApJ, 347, 529

Wang, Y.-M., Sheeley, N. R., \& Nash, A. G. 1991, ApJ, 383, 431

Wang, Y.-M., \& Sheeley, N. R. 2013, ApJ, 764, 90

Weiss, N. O., Cattaneo, F., Jones, C. A. 1984, Geophys. Astrophys. Fluid Dyn., 30, 305

Wilmot-Smith, A. L., Martens, P. C. H., Nandy, D., Priest, E. R., \& Tobias, S. M. 2005, MNRAS, 363, 1167

Yeates, A. R., Nandy, D., \& Mackay, D. H. 2008, ApJ, 673, 544

Zhao, J., Nagashima, K., Bogart, R. S., Kosovichev, A. G., \& Duvall, T. L. 2012, ApJ, 749, L5 